\title{
SIOP/COG/NWTSG Stage II
}

National Cancer Institute

\section{Source}

National Cancer Institute. SIOP/COG/NWT SG Stage II. NCI Thesaurus. Code C140303.

Wilms' tumor extends beyond the kidney but is completely excised; no residual tumor is apparent at or beyond the margins of the excision; tumor thrombus in vessels outside the kidney is Stage II if the thrombus is removed en bloc with the tumor. 